\title{
User interface evaluation of serious games for students with intellectual disability
}

Cecilia Sik Lanyi ${ }^{1}$, David J. Brown ${ }^{2}$, Penny Standen ${ }^{3}$, Jacqueline Lewis ${ }^{4}$ and Vilma Butkute ${ }^{5}$

1 Virtual Environment and Imaging Technologies Laboratory, Department of Electrical Engineering and Information System, University of Pannonia, Egyetem u. 10., H-8200 Veszprem, Hungary

${ }^{2}$ School of Science and Technology, Nottingham Trent University, Clifton Campus, Clifton Lane, Nottingham NG11 8NS, UK

${ }^{3}$ Division of Rehabilitation and Ageing, School of Community Health Sciences, University of Nottingham. B Floor, Medical School, QMC. Clifton Boulevard, Nottingham NG7 2UH, UK ${ }^{4}$ Greenhat Interactive Ltd, 6 Church Street, Kidderminster, DY10 8AD, UK

${ }^{5}$ Vilnius University, The Faculty of Philosophy, Education department, Universiteto str. 9/1, Vilnius, Lithuania,

\{lanyi@almos.uni-pannon.hu, david.brownentu.ac.uk, p.standen@nottingham.ac.uk, jacqui.lewis@virgin.net, vilma.butkutedimotec.lt\}

\begin{abstract}
We have designed and evaluated around 10 serious games under the EU Leonardo Transfer of Innovation Project: Game On Extra Time (GOET) project http://goet-project.eu/. The project supports people with learning disabilities and additional sensory impairments in getting and keeping a job by helping them to learn, via games-based learning; skills that will help them in their working day. These games help students to learn how to prepare themselves for work, dealing with everyday situations at work, including money management, travelling independently etc. This paper is concerned with the potential of serious games as effective and engaging learning resources for people with intellectual disabilities. In this paper we will address questions related to the design and evaluation of such games, and our design solutions to suit the individual learning needs of our target audiences.
\end{abstract}

Keywords: intellectual disability, serious games, user interface testing

\section{Introduction}

We have designed and evaluated around 10 serious games under the EU Leonardo Transfer of Innovation Project: Game On Extra Time (GOET) project. The project supports people with learning disabilities and additional sensory impairments in getting and keeping a job by helping them to learn, via games-based learning; skills that will help them in their working day. These games help students to learn how to prepare themselves for work, dealing with everyday situations at work, including money management, travelling independently etc. In this paper we will address 
questions related to the design and evaluation of such games, and our design solutions to suit the individual learning needs of our target audiences. It is necessary to design the user interfaces for maximum accessibility and usability. In this way we will minimise the additional cognitive load placed on the user while navigating within the software. In order to achieve these goals we have followed published design guidelines, and placed emphasis on using graphics, animations, interactivity, choice and auditory output to promote user engagement and provide alternatives to text. In this paper we will address the pilot testing of the user interface of these serious games.

These games which are tested in all partner countries (UK, Lithuania and Hungary) include [1]:

- 3D Work Tour: simulating the first days at work in a games 'mod' created using the Half Life 2 engine

- Cheese factory: teaching the students using fractions and percentages based on the popular Tetris Game.

- Memobile: trains the student in the important things to do in preparing to leave the house and throughout their working day using mobile phone technology programmed using Flash.

- My Appearance: covering everyday routines such as personal hygiene and getting ready for work-tasks from getting up until leaving home using a Flash game.

- VR supermarket: helps to teach students about money management skills within a store environment developed using Flash.

\section{Description of the tested serious games}

In this section the main design requirements of the tested games: " $3 \mathrm{~d}$ Work Tour", "Cheese factory", "Memobile", "My Appearance", and VR supermarket" game are described.

\subsection{D Work Tour}

3D Work Tour: simulates the first days at a workplace in a games 'mod' created using the Half Life 2 engine. After selecting the language there are two possibilities: subtitles and video tour with BSL (British Sign Language) for hearing impaired users. The user interface is very simple and very clearly organised. The VR environment and avatars are realistic and look similar to the work-based environments and people in the real world (Fig 1 and Fig 2). 


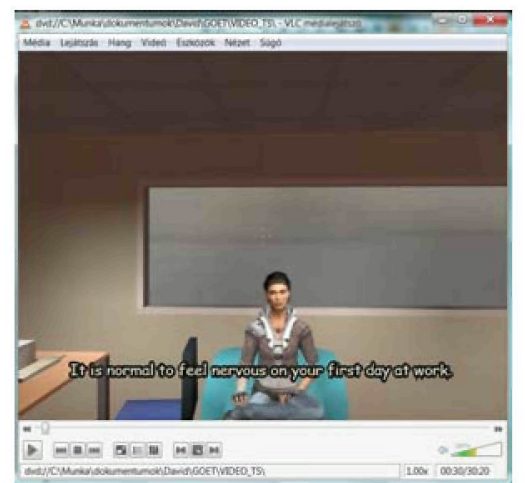

Fig. 1. Using subtitles and sound files

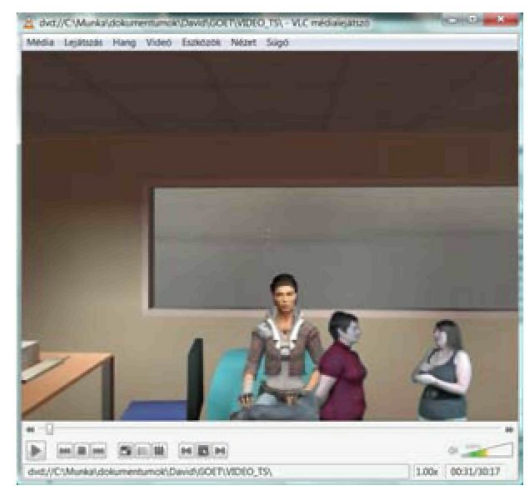

Fig. 2. Using BSL

\subsection{Cheese factory}

Cheese factory: teaches the students using fractions and percentages based on the popular Tetris Game. The user interface of this game is simple too. The instructions are clear, the colours are appropriate, and they are in harmony with the overall interface. The users' results are shown on the right side of the game and the next piece of the cheese is also shown. These features support the user in their ongoing learning tasks (Fig 3 and Fig 4).

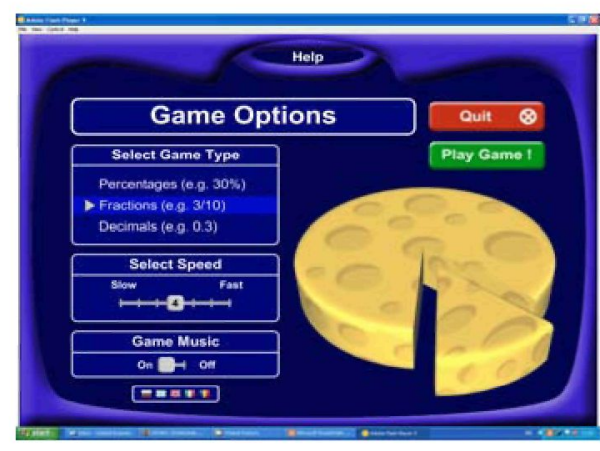

Fig. 3. Options of the game

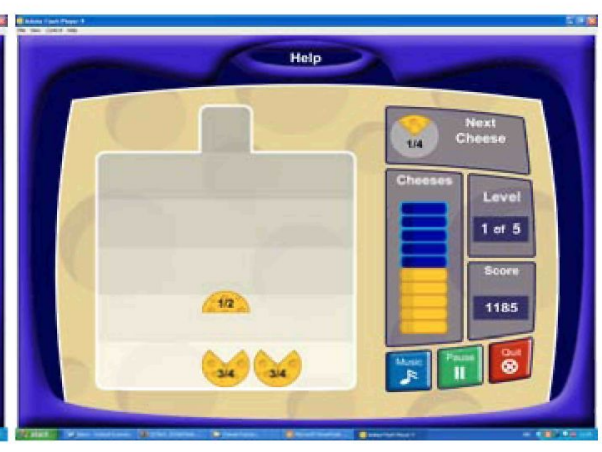

Fig. 4. Showing the process of the game

\subsection{Memobile}

The Memobile game trains the student in the important things to do in preparing to leave the house and throughout their working day using mobile phone technology programmed using Flash. 


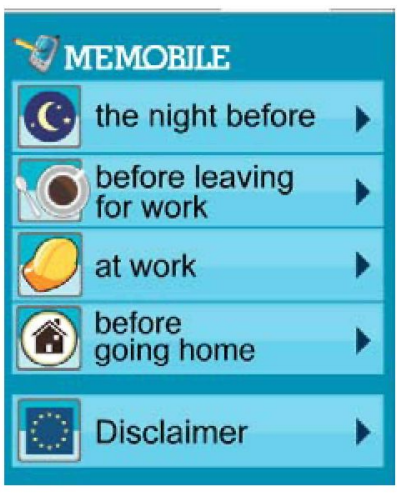

Fig. 5. Main menu of the "Memobile" game

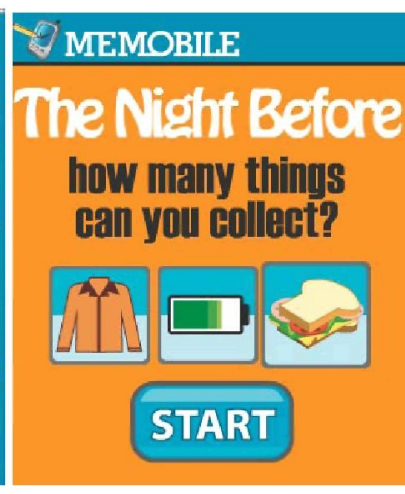

Fig. 6. Solving one task

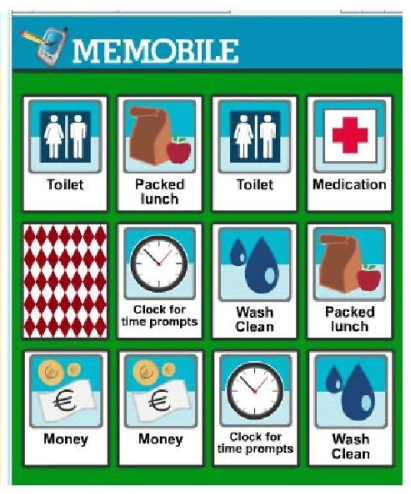

Fig. 7. Memory in the "Memobile" game

\subsection{My Appearance}

My Appearance: teaches the students' everyday "morning" tasks from getting up until leaving home using a Flash game. The graphic interface of the game is clear and understandable, and cartoon-like. It simulates the sequencing of morning tasks in preparation for leaving for work, and the structure of the game is very consistent. For example after getting up, after having a shower, getting dressed (Fig. 8) and eating breakfast (Fig 9), the user's avatar is ready to leave for work and its appearance improves (Fig 10).

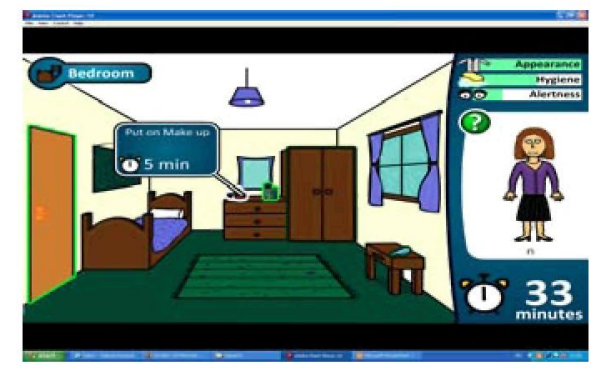

8. After having a shower and getting dressed

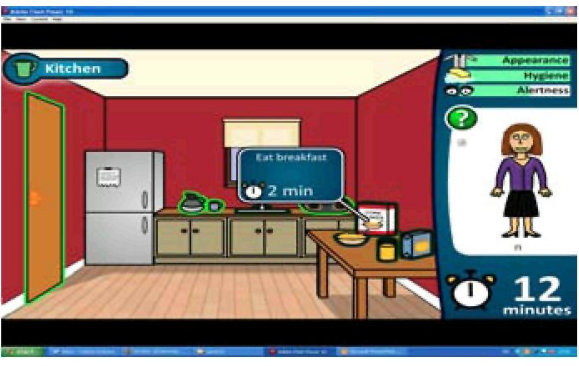

Fig. 9. Eating breakfast 


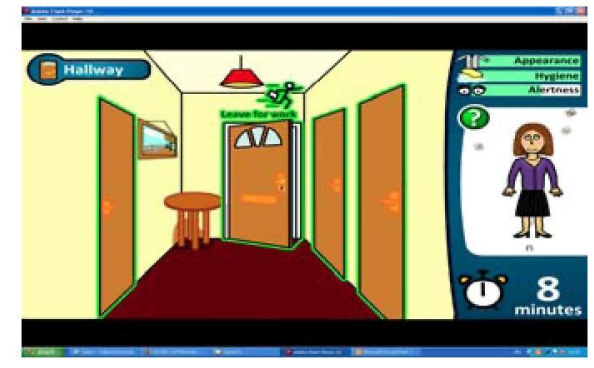

Fig. 10. Ready for leaving home

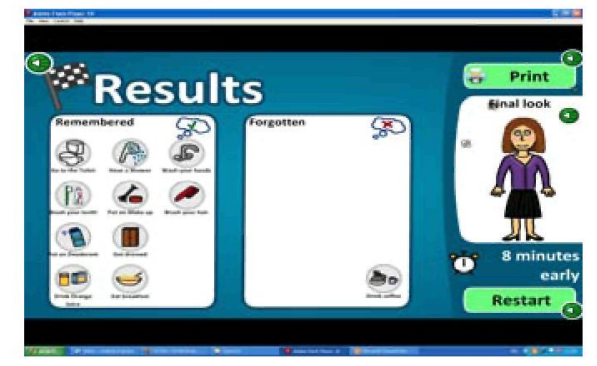

Fig. 11. Showing the results graphically

At the end of the game the user receives feedback on his/her performance using sound, subtitles or BSL. If the student forgets to wash his/her hands or forgets to have a morning drink, the game doesn't interfere - it lets the student make mistakes and learn from doing so by reflecting on the game responses to their actions (Fig 11).

\subsection{VR supermarket}

VR supermarket game helps to teach students about money management skills within a store environment developed using Flash. The player enters the virtual supermarket (Fig 12), and is given a virtual wallet, shopping list (Fig 13) and shopping cart.

The goods on a given shelf are displayed with their names, prices and images attached to them. To place an item to the shopping cart, the player only has to click on the given item (Fig 14).

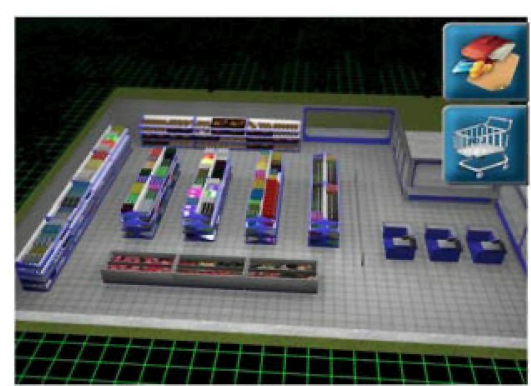

Fig. 12. VR supermarket

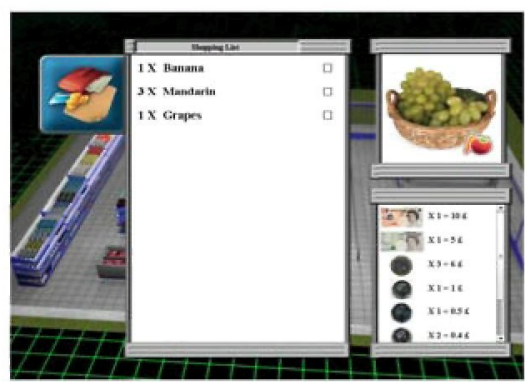

Fig. 13. Shopping list

Before paying, the bar code scanner registers the price of each item in the shopping cart one by one. During this both the cashier and the cash register will give feedback to the student (Fig 15). To pay for the items the student has to place a sufficient sum of money onto the drop panel by clicking the separate banknotes and coins in the wallet and then hitting the pay button. After payment the cashier gives change if necessary, and the "go home" button appears to finish the task. If the student has a insufficient amount of money or has forgotten something, the "back" button leads the 
user back into the store. Clicking on the "help" button reveals a small panel on which the paid amount of money is shown.

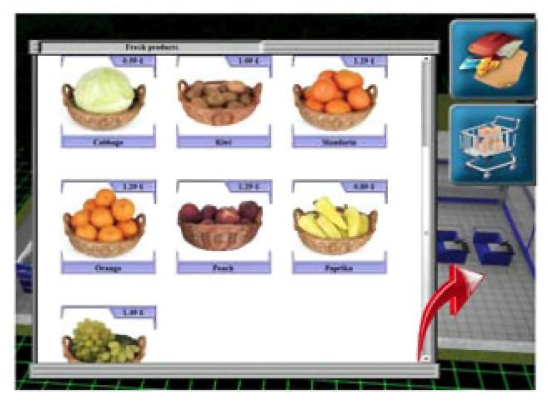

Fig. 14. Goods on a shelf

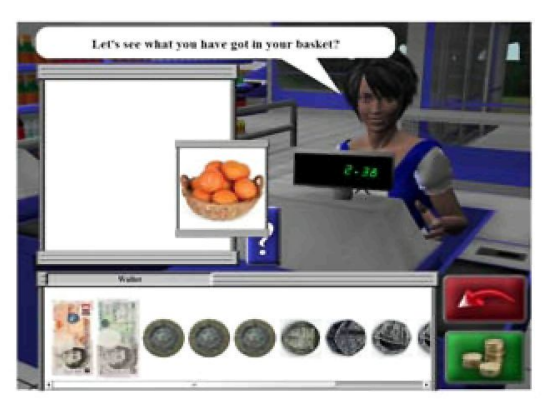

Fig. 15. At the cash register

\section{Evaluation of the user interface}

We have developed a 5 point Likert Scale close-ended questionnaire for testing the user interface of the newly developed serious games.

Likert Scales: Likert scales are scales on which the participants register their agreement or disagreement with the statement. "_Strongly Disagree, _ Disagree, _Neither Agree nor Disagree, _ Agree,_Strongly Agree" on a five-point scale.

This questionnaire contains 29 questions arranged in 4 themes:

\subsection{To what degree are the games enjoyable? (6 questions)}

How engaging are the games?

- Using the software was tiring.

- The content of the software was engaging.

- Playing with the software was boring.

- The games were easy to play.

- Activities in game play were predictable.

- I used the software willingly.

\subsection{Questions concerning the usability of the software (4 questions)}

Questions regarding the usability of the software:

- The software displays realistic situations.

- The presented situations were relevant and important.

- Are you satisfied with the quantity and diversity of the questions used?

- Using the software was easy. 


\subsection{Questions concerning the software's manageability: ( 9 questions)}

Questions regarding the software's manageability:

- There is sufficient opportunity to correct or revisit responses given in the game

- The content areas between levels are distinct.

- It was easy to follow the activities in the game.

- The noises or music used in the games were disturbing.

- The speech used was clear and understandable.

- Failures were typically results of disorientation.

- Starting the software was an easy process.

- Closing the software/Quitting at the end of the game was easy.

- Quitting during the game was easy.

\subsection{Questions concerning the graphics (10 questions)}

Questions regarding the graphics:

- The software's look is realistic.

- The software's look is likeable.

- The software's look was appropriate to its aim.

- The pictures used were easy to recognize.

- The illustrations and backgrounds used were helpful.

- The connection between the pictures and the actions rendered to them were unambiguous.

- Failures were typically results of not being able to recognise pictures.

- Failures were typically results of not understanding instructions.

- The software was rich in (visual and audio) stimuli.

- The screens of the software were detailed.

After every question there is a "If you wish comment, why you have selected the given answer" open ended question to supplement the response given. At the end of the test there is a " $30^{\text {th }}$ question" - a half page for further notes, comments and remarks.

\subsection{Further testing}

In a mixed methods approach the questionnaires are complemented by two further tools suitable for testing accessibility and usability early on in the implementation process. The first is an Expert Review Tool using usability heuristics (guidelines for good design) selected by the multivariate design team (project steering group) against which the games will be assessed in group project meetings (two passes of the software). The second is an Observational Checklist for use by the evaluation researcher to record data in categories including accessibility, usability, engagement, unsolicited comments, and help required (actions and verbal prompts) whilst users are trialling the serious games. 


\subsection{Design principles}

We tested the following serious games: 3D Work Tour, Cheese factory, Memobile, My Appearance and VR Supermarket software. Based on the evaluation results we iterated our own design guidelines for serious games for use by people with intellectual and additional sensory impairments which can currently be summarised as [1], [2]:

- Ensure presentation at appropriate speed

- Allow users to go back

- Allow User Control

- Make any text plain text

- Never convey information by colour alone

- Ensure sufficient contrast

- Help users navigate

- Make clear Maintain organisation

- Use unique and informative text descriptions for any hyperlinks (never click here!)

- Use accessibility features

- Design simply in simple layouts

- Use fallbacks

- Make systems consistent and error free

- Aim for compatibility with assistive technologies

- Allow keyboard access

- Do not include elements that are known to cause seizures

\section{Conclusion}

In this paper we have discussed the design and evaluation of five serious games' and their user interfaces. These serious games were developed for students with intellectual disability to help them in activities of daily living, their working life and specifically in managing a budget. We have demonstrated the test process of the user interface design of the serious games and our solutions for any identified problems for students with intellectual disability. The detailed pedagogical tests will run until July 2010.

Acknowledgments. This project has been funded with support from the EU's Transfer of Innovation Leonardo da Vinci Lifelong Learning Programme, Game on Extra Time, GOET Grant Agreement number: UK/08/LLP-LdV/TOI/163_181 for.

\section{References}

1. Sik Lanyi, C., Brown, D.J.: Design of serious games for students with intellectual disability, India HCI 2010, Interaction Design for International Development 2010, Bombay, India, March 20-24. 2010, pp. 151-160.

2. Brown D.J., Standen P., Evett L., Battersby S., Shopland N.: Designing Serious Games for People with Dual Diagnosis: Learning Disabilities and Sensory Impairments. In Press in Educational Gaming. IGI Global, editors Pavel Zemliansky; Diane Wilcox. 